\title{
Systematic Analysis of Virtual Reality \& Augmented Reality
}

\author{
Asif A. Laghari* \\ Department of Computer Science, Sindh Madressatul Islam University, Karachi, Pakistan \\ Email: asif.laghari@smiu.edu.pk

\section{Awais K. Jumani, Kamlesh Kumar, M. Ameen Chhajro} \\ Department of Computer Science, ILMA University Karachi, Sindh, Pakistan \\ Department of Computer Science, Sindh Madressatul Islam University, Karachi, Pakistan \\ Email: awaisjumani@yahoo.com, kamlesh@ smiu.edu.pk, amen.chhajro@smiu.edu.pk
}

Received: 19 October 2020; Accepted: 20 January 2021; Published: 08 February 2021

\begin{abstract}
Nowadays, users are moving from old 2D screens to modern devices such as 3D screens and virtual reality devices to enjoy videos and games like real-world experience, and this demand increased further development. Virtual Reality (VR) is based on the creation of a simulated environment of real-world with computer creation, and Augmented Reality (AR) is based on the addition of simulation components (environment) in the real-world scene. In this paper, systematic analysis of relationships and features both VR and AR varies by outline, arrangement, administrations, and devices for associations and clients. This paper provides a difference between AR and VR, advantages, future, and open research issues.
\end{abstract}

Index Terms: Virtual Reality, Augmented Reality, Digital environment

\section{Introduction}

Virtual Reality (VR) is characterized as the utilization of computer innovation to form a simulated environment [1]. After you see VR, you are watching a distinctive reality than the one before you. Virtual reality may be artificial, such as an enlivened scene, or a real put that has been shot and included in a virtual reality app.

Augmented reality is characterized as an improved form of reality made by the utilization of innovation to include computerized data on a picture of something [2]. AR is utilized in apps for smartphones and tablets. AR apps utilize your phone's camera to appear you a set of the real world before you, at that point put a layer of data, counting the content and/or pictures, on top of that view.

The idea of augmented reality (AR) first applied in mid-1980 by Jaron Lanier, who was the planner of VPL Research [3]. He started to build equipment based on the computer-generated reality such as goggles and gloves. Indeed, even before that, nevertheless, scientists were making recreated conditions. One accomplishment was the Sensorama in 1956. Another researcher named Morton Heilig was involved in the research of the perception that how the user will feel that he is in the movie during the watching [4]. The Sensorama experience reinvented a genuine city condition, which you "rode" through on a cruiser. Multisensory stimulation lets you realize the street, feel the vibration, hear the motor, and smell the engine's fumes in the structured "world".

Heilig also protected a head-mounted showcase device, called the Telesphere Mask, in 1960. This work expended further and in 1965 Ivan Sutherland developed "the Ultimate Display," a head-mounted device that he proposed would fill in as a "window into a virtual world [5]." VR/AR research rapidly developed in the 1970s and 1980s. In the mid1980s at NASA Ames Research Center, the Virtual Interface Environment Workstation (VIEW) framework consolidated a head-mounted gadget with gloves to empower the haptic connection.

The present life pattern of augmented reality may have started when the most punctual models of the Oculus Rift appeared at the E3 videogame public expo in 2012; however, it's been licking at the edges of our aggregate cognizance for over a century [6]. Immersing ourselves in 3D situations dates right back to the stereoscopes that enraptured individuals' minds in the nineteenth century. On the off chance that you present a practically indistinguishable picture to each eye, your cerebrum will consolidate them and discover profundity in their errors; it's a similar component ViewMasters used to turn into a youth staple. Fig. 1 provided facts of evolution of VR/AR technology from start to till now [19]. 
The users of non-development countries are still using old 2D screens for watching videos. 3D devices are costly for common users, however, 3D videos increase the user experience, enjoyment, and satisfaction level of users when they play games or watch videos. Further, VR increases the user experience when 3D videos were played or viewed on VR devices. The AR also increases the enjoyment when the developer/user adds new things in videos or games. Still, $\mathrm{AR}$ in the development phase, and future technologies were common for all users.

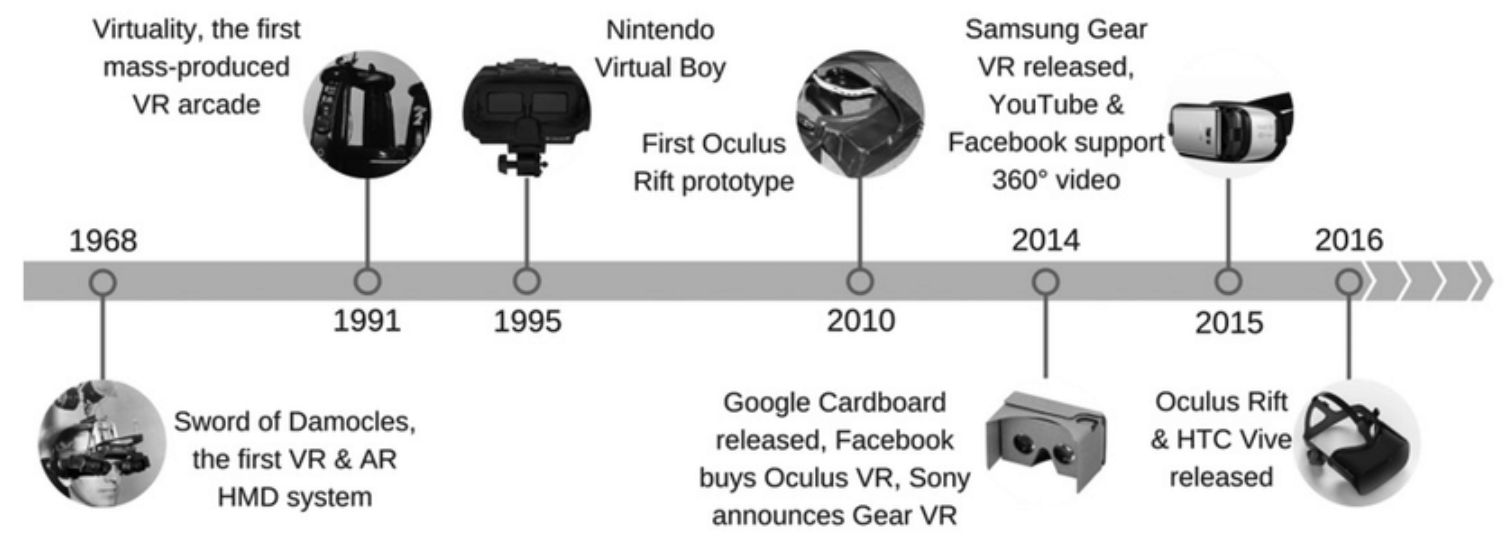

Fig. 1. Evolution of VR/AR technology

\section{A. Stereoscopic Display}

The stereoscopic display is essential for VR filmmaking. This technique is referred for making a sense of depth for an image that creates each eye focus in harmony. The word originates from the Greek word $\sigma \tau \varepsilon \rho \varepsilon$ ó $\varsigma$ (stereos), which means "solid" and бко $\varepsilon \dot{\varepsilon} \omega$ (skopeō), meaning "to see."

The vision system of humans is based on the merging of two viewpoints from the left and right eye-into a single image. To control, dimension, distance, and speed the multiple zone input of eyes allows the brain to execute computations. Similarly, to increase watching experience with multiple dimensions and depth to give information to each eye, the stereoscopic video is designed. Fig. 2 shows view of anaglyph and polarized 3D glasses [7].

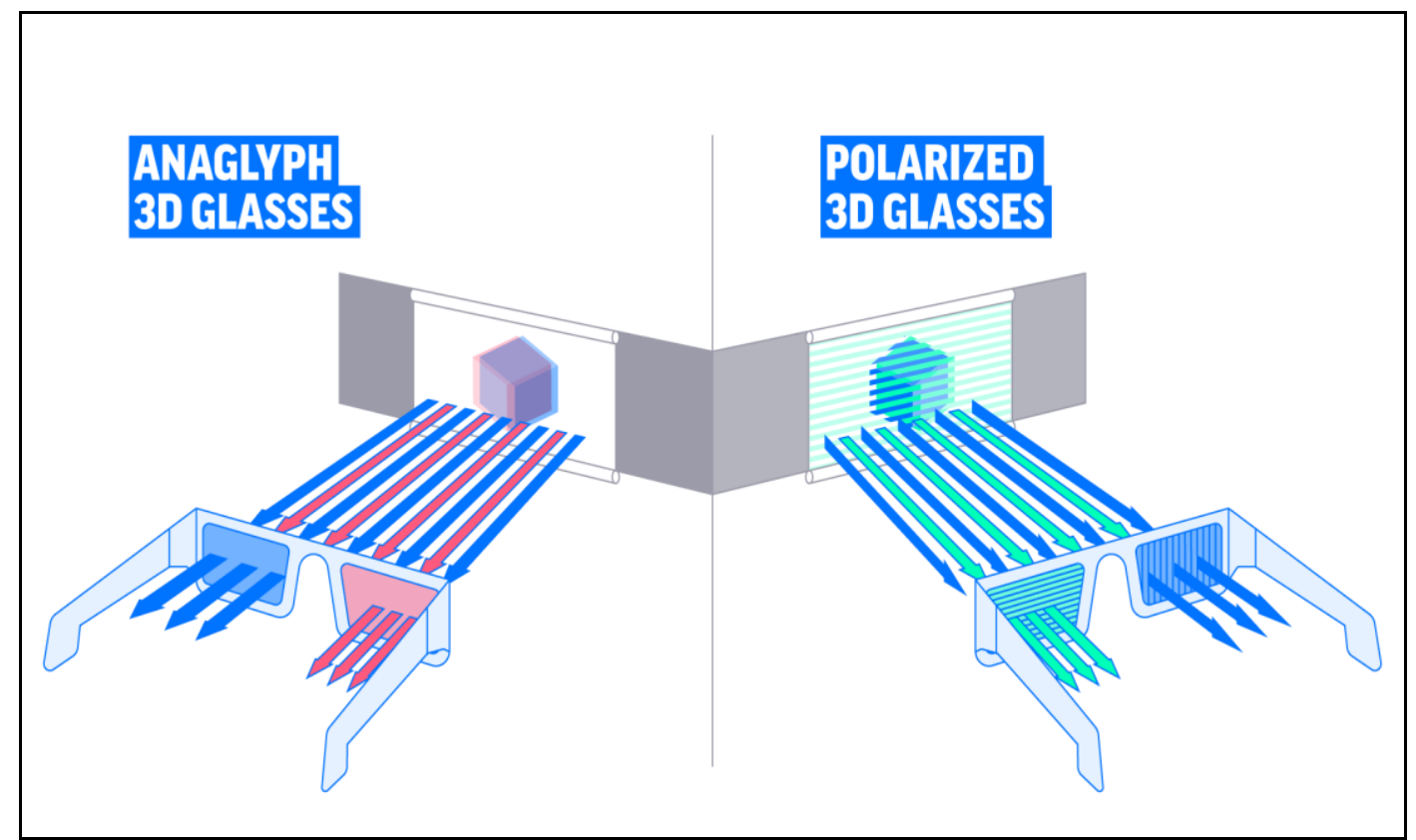

Fig. 2. View of anaglyph and polarized 3D glasses 


\section{B. Tracking Motion}

Tracking the user of the head, and even their whole body, permits them to be put inside the 3D designs, and this implies they can encounter certifiable special awareness because of right review parallax and change of point of view during development [8].

\section{Input Devices}

Input devices are mostly used in joysticks, Tracking balls, force balls, data gloves, track pads.

\section{Virtual Reality Headset}

A (VR) Headset is a head-mounted device that gives virtual reality to the wearer. Virtual reality (VR) headsets are mainly used with computer games yet they are also used in different applications including test systems and coaches [9].

\section{E. Virtual Reality Types}

There are three fundamental sorts of Virtual reality utilized nowadays to change our general surroundings, including non-immersive, semi-immersive, and fully-immersive simulations. To show signs of improvement comprehension of how the innovation is utilized, how about we separate the various sorts of VR and see instances of each [10].

The paper will address the topic of VR and AR, the start of art, definitions, and how VR and VR are providing the experience of viewing videos the same as the real world. Further, we provide details of the difference between them, challenges to design according to user needs, limitations. Finally, we provide open research for future research direction, which will never be discussed.

This paper is organized into

\section{Difference Between Virtual Reality and Augmented Reality}

The term Virtual reality and augmented reality, they sound similar and if you see their technologies develop, they seem over into each other a bit. They are two very different technologies and their concepts with the help of characteristics that readily distinguish them from one another [11]. Figure 3 show the VR is complete digital environment and AR real world scene presented with addition of digital information, illusion is given Fig.3 [20].
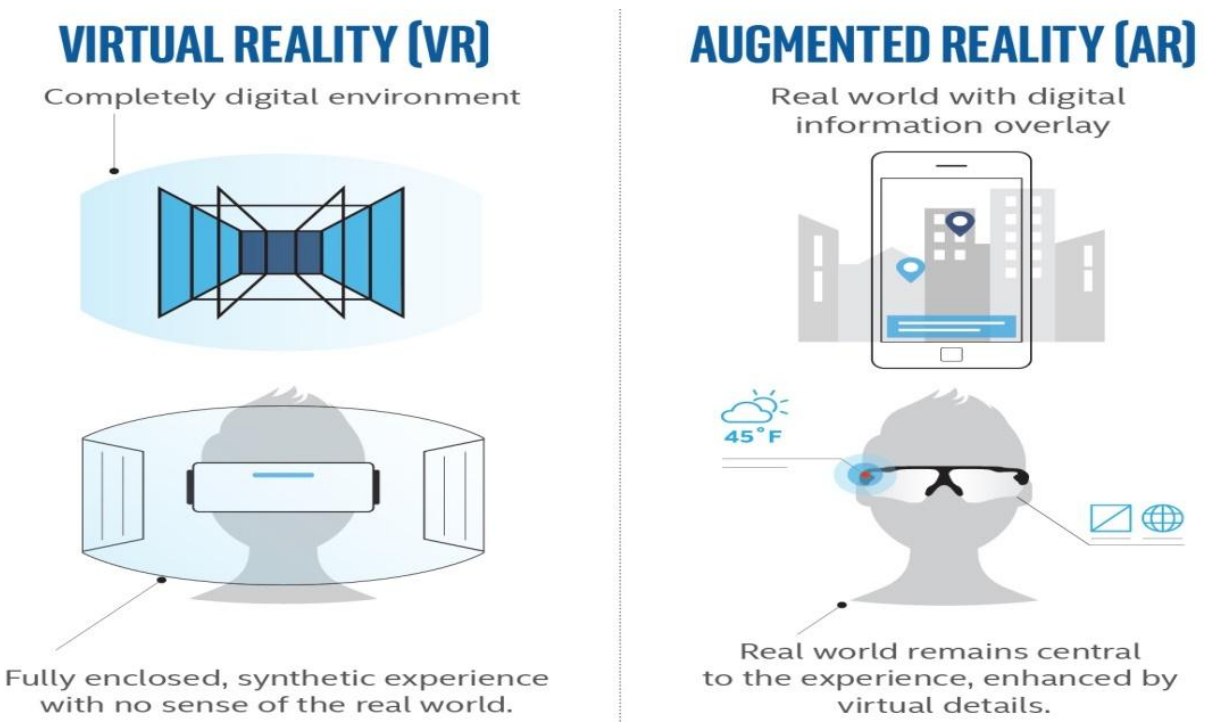

Fig. 3. Presentation of AR and VR to audience

A lot of transformative technologies keep coming and our lifestyle changes our daily routine. With such capacities, we can see that VR and AR are holding the power to change the future of facing world challenges. They are going to change the way we look at the world. These technologies are changing many of the current means of entertainment so it is the most basic condition that we should be able to separate them. A comparison of features and differences are given in Table 1. 
Table 1. Difference between AR and VR

\begin{tabular}{|c|c|c|}
\hline & AR & VR \\
\hline Photo Real & No & No \\
\hline 3D Asset Type & Low/Medium Poly & Low/Medium Poly \\
\hline Primary User & Consumers & Consumers \\
\hline Ideal Channel & Online & In-Store \\
\hline Expected ROI & Increased Conversions & High \\
\hline Investment Cost & Medium & Immersive virtual shop experiences \\
\hline Top Use Case(s) & Visualizing virtual world products in a real environment
\end{tabular}

A. $\quad V R$ :

- Where VR is replacing our vision.

- VR is considered as a complete imagination experience that shut out the physical world.

- Users can move from the real world to a virtual imagination environment by wearing a special headset. These headset devices are HTC Vive, Oculus Rift, or Google cardboard, which is very expensive [12].

- For both games and apps, VR completely changed our surrounding environment and take us to other places. Where we are physically it doesn't matter.

- VR is $25 \%$ real and $75 \%$ virtual.

- By using VR technology users are completely secluded from the real world and enter him into the imagination or fictional world.

B. AR:

- Augmented reality adds to it

- On the other hand, augmented reality ads 3-D digital elements or objects to our live view means in the real world.

- Augmented reality used the camera on my smartphone to add or place an object for example augmented reality and experiences include Snapchat lenses and filters and the game Pokémon Go, which we had already discussed [13].

- This tech has a distinct disadvantage compared with virtual reality because augmented reality apps only show up on users' smartphone or tablet screen and can only project images in a limited area in front of his eyes.

- AR is $25 \%$ virtual and $75 \%$ real.

\section{Advantages and Disadvantages}

Virtual Reality also some advantages like increase capacity to work and virtual reality work in the field of education where helps students to understand the historical places as they were there at the time of happening. Despite many advantages, there are also so many disadvantages for instance, if workers getting training in a virtual environment have never the similar outcome as training in the real world because there is no assurance that a person doing well in the real world.

Also, Augmented Reality has so many advantages like anybody can learn individually and increase their innovation and improvement in every wide variety of fields and help creators to develop games that offer a real experience to the use. Despite many advantages, there are also many disadvantages like it is very expensive to implement and develop the AR technology-based project and maintain it because of lack of privacy issues is also a major drawback in the AR technology.

\section{The Future of VR and AR}

What this is for is an inquiry that does not have a solitary answer. The simplest yet least fulfilling reaction is that it is for everything. Past games and other intuitive amusement, VR shows promising applications for relief from discomfort and PTSD, for instruction and plan, for both working from home and office work [14]. On account of "encapsulated nearness" you involve a symbol in virtual space, social VR is not simply more vivid than any carefully interceded correspondence we have at any point experienced, however all the more influencing also. The encounters we have, from our responses to our environmental factors to the nature of our communications, are put away and recovered in our cerebrums like some other experiential memory.

That may wind up originating from Apple; the Cupertino organization is busy working on a headset that could dispatch as ahead of schedule as 2020. Be that as it may, staggeringly all around supported and much more unimaginably mysterious organization Magic Leap has as of late rose up out of long stretches of watched advancement to dispatch the primary designer just form of its AR headset; the organization has said its gadget would have the option to convey conventional VR just as a multi-dimensional image-driven blended reality. 
However, even with that kind of device, we are toward the start of a long, unsure street not due to what the innovation can do, but since of how individuals could abuse it. The web is incredible; how individuals treat each other on the web, not really. Apply that rationale to VR, where being exemplified as a symbol implies you have individual limits that can be disregarded, and where specialized sound and haptic criticism allows you to hear and feel what others are stating and doing to you, and you are taking a gander at a potential for badgering and dangerous conduct that is exponentially more instinctive and damaging than anything on regular internet based life.

When AR finds a convincing, full-included stage, and it turns out to be evident that an immense number of customers are turning out to be AR capable, the capability of AR will start to be completely figured out. Each industry from education to architecture, military training, Sports, and selling business will profit by accepting AR [15].

Different industries organized by Total Immersion ${ }^{\mathrm{TM}}$ that will see expanded AR movement sooner rather than later in its t-immersion.com blog entry, "The Future of Augmented Reality." These enterprises include:

- E-Commerce: In the future, several organizations will add AR features into mobile apps on their websites. In retail, this will result in applications that seamlessly "clothe" a user in footwear, jewelry, jackets, and sunglasses via the camera in the person's smartphone.

- Digital Marketing: AR innovations will keep on improving how clients engage in with brands. Promoting AR will probably be found in Wrapping, Interactions with different items, through gaming applications, and street signs.

- Geo-location: The capacity of cell phones to illuminate us regarding our environmental factors is extraordinarily enhanced over time. AR could assistance everything from ongoing tourism warnings to café recommendations.

- Educational Resources: Researchers are as of now endeavoring to discover new and useful approaches to utilize AR in preparing circumstances. The military and medicinal services enterprises, specifically, are emerging powerful AR training simulations.

\section{Open Research Issues:}

\section{A. User protection}

Due to the nature of headset physical protection of end-user required if it depends on the real-time sensory feedback user will stop walking on the walls or neglect to perceive key threats in their prompt environmental factors [16]. In this regard, some resolutions are suggested including using a circular walking arc however; it still needs to require more research to efficient.

\section{B. Pornographic content}

How to avoid pornography is a big issue in the usage of VR/AR technologies [17]. This is also evidence that extreme publicity to pornography could influence harmful behavior toward women. If clients increase the usage of pornographic content in a new realistic environment with a first-person style of interaction, what effects could that have on violent crime? This will be a big problem if virtual sex contents simulated real-world persons and this is an illegal act according to the real-world rules.

\section{User isolation and social effects}

A VR/AR gaming increase interest and attracts more users to play games, and this technology provides a more realistic environment to enjoy games so; users continue to play games [18, 21, 22]. In this situation, the user is isolated from the world and social distances will increase and also harmful for health. So there is also research work required to avoid this situation if the entire immersive world is available to explore then that will stop to happing this [23].

\section{Real-world applications}

If the user is always using virtual content, then it will difficult for the user to return to the real world and act in the same way as they did before virtual experience. This will damage the social relationship of the user with the real world and also a certain type of insensitive interaction or violence. Research required avoiding situations happing before promote and further development of VR/AR [24].

Users now are moving from 2D to 3D video with VR devices, however, they face problems because heterogeneous VR devices of different organizations provide low and high user satisfaction sense of watching of videos [25, 26, 27]. The researchers did not use the quality of experience (QoE) assessment for VR devices using different parameters such as graphics quality, and the impact of network condition for online videos [28, 29, 30]. QoE assessment of VR videos was never considered for research purposes so still research work required assessing the user needs for AR videos, which will improve the user experience for AR technology [31, 32]. VR/AR gaming is also an emerging research area; there is a need for assessment of gaming quality from VR devices to get user requirements for the future development of VR devices [33, 34]. 


\section{Conclusion}

In this paper, we presented a systematic analysis of VR and AR, which will help researchers to recognize the difference between both technologies. A lot of research work has done on VR, and now it is mature, AR still under development phase and researcher combining both to develop mixed immersive. We believe that VR/AR and mixed immersive is the future of the new multimedia revolution, and this will be used in a different field in the future. The Table given in the paper provides details of comparison and advantages. Immersive technology will be promising business models for service providers in the future.

\section{Conflict of Interest}

Authors did not have any conflict of interest.

\section{References}

[1] Hodgson, Paula, V. WY. Lee, J. CS. Chan, A. Fong, C.SY.Tang, L.Chan, and C.Wong. "Immersive virtual reality (IVR) in higher education: Development and implementation." In Augmented Reality and Virtual Reality, pp. 161-173. Springer, Cham, 2019.

[2] Alalwan, Nasser, L. Cheng, H. Al-Samarraie, R. Yousef, A. I. Alzahrani, and S. M. Sarsam. "Challenges and Prospects of Virtual Reality and Augmented Reality Utilization among Primary School Teachers: A Developing Country Perspective." Studies in Educational Evaluation 66 (2020): 100876.

[3] Faisal, Aldo. "Computer science: visionary of virtual reality." Nature 551, no. 7680 (2017): 298.

[4] Jamali, S. Salmi, M. F. Shiratuddin, and K. W. Wong. "A review of augmented reality (AR) and mobile-augmented reality (mAR) technology: Learning in tertiary education." Learning in Higher Education 20, no. 2 (2013): 37-54.

[5] Stec, Jacob, and S. K. Shanmugam. "A Study and Implementation of Virtual Reality and its Capabilities." In Proceedings of the 2020 the 4th International Conference on Compute and Data Analysis, pp. 203-207. 2020.

[6] Roettl, Johanna, and R. Terlutter. "The same video game in 2D, 3D or virtual reality-How does technology impact game evaluation and brand placements?." PloS one 13, no. 7 (2018).

[7] Retrieved from: https://courses.vrtl.academy/lessons/whats-up-with-stereoscopic-and-virtual-reality/, 2020

[8] Ng, KT. Adrian, L. KY. Chan, and H. YK. Lau. "A study of cybersickness and sensory conflict theory using a motion-coupled virtual reality system." Displays 61 (2020): 101922.

[9] Weech, Séamas, S. Kenny, M. Lenizky, and M. Barnett-Cowan. "Narrative and gaming experience interact to affect presence and cybersickness in virtual reality." International Journal of Human-Computer Studies 138 (2020): 102398.

[10] Buttussi, Fabio, and L. Chittaro. "Effects of different types of virtual reality display on presence and learning in a safety training scenario." IEEE transactions on visualization and computer graphics 24, no. 2 (2017): 1063-1076.

[11] Li, Xiao, W. Yi, H.L. Chi, X. Wang, and A. PC Chan. "A critical review of virtual and augmented reality (VR/AR) applications in construction safety." Automation in Construction 86 (2018): 150-162.

[12] Siriborvornratanakul, Thitirat. "A study of virtual reality headsets and physiological extension possibilities." In International Conference on Computational Science and Its Applications, pp. 497-508. Springer, Cham, 2016.

[13] Paavilainen, Janne, H.Korhonen, K. Alha, J. Stenros, E. Koskinen, and F. Mayra. "The Pokémon GO experience: A locationbased augmented reality mobile game goes mainstream." In Proceedings of the $2017 \mathrm{CHI}$ conference on human factors in computing systems, pp. 2493-2498. 2017.

[14] Mishkind, Matthew C., A. M. Norr, A. C. Katz, and Greg M. Reger. "Review of virtual reality treatment in psychiatry: evidence versus current diffusion and use." Current psychiatry reports 19, no. 11 (2017): 80.

[15] Yung, Ryan, and C. Khoo-Lattimore. "New realities: a systematic literature review on virtual reality and augmented reality in tourism research." Current Issues in Tourism 22, no. 17 (2019): 2056-2081.

[16] Riscombe-burton, Phillip. "AR/VR device virtualisation." U.S. Patent 10,290,151, issued May 14, 2019.

[17] Slater, M., C. Gonzalez-Liencres, P. Haggard, C. Vinkers, R. Gregory-Clarke, S. Jelley, Z. Watson et al. "The Ethics of Realism in Virtual and Augmented Reality. Front." Virtual Real. 1: 1. doi: 10.3389/frvir (2020).

[18] Saunders, Cariann. "The Oculus Rift and the Human Psyche: The Societal Effects of Virtual Reality and Its Accompanying Immediacy." The Classic Journal (2016).

[19] https://app.emaze.com/@ AOFWWIZCR\#5 [accessed on 12/09/2020]

[20] https://draup.com/sales/insights/retail-brands-ar-vr-solutions-brand/ [accessed on 12/09/2020]

[21] Laghari, Asif Ali, Kamran Ali Memon, Muhammad Bux Soomro, Rashid Ali Laghari, and Vishal Kumar. "Quality of experience (QoE) assessment of games on workstations and mobile." Entertainment Computing (2020): 100362.

[22] Laghari, Asif Ali, Hui He, Kamran Ali Memon, Rashid Ali Laghari, Imtiaz Ali Halepoto, and Asiya Khan. "Quality of experience (QoE) in cloud gaming models: A review." Multiagent and Grid Systems 15, no. 3 (2019): 289-304.

[23] Alcabaza, Dan Vincent G., Marvin E. Legaspi, Trisha Leinelle Muyot, Kate Louise C. Ofren, Johsua Angelo D. Panganiban, and Roselito E. Tolentino. "Real-Time Realistic Telepresence using a 360 Camera and a Virtual Reality Box." (2019).

[24] Yong, Sei Wei, and Aun Naa Sung. "A Mobile Application of Augmented Reality for Aircraft Maintenance of Fan Cowl Door Opening." International Journal of Computer Network and Information Security 11, no. 6 (2019): 38.

[25] Arora, Rahul, Rubaiat Habib Kazi, Tovi Grossman, George Fitzmaurice, and Karan Singh. "Symbiosissketch: Combining 2d \& $3 \mathrm{~d}$ sketching for designing detailed 3d objects in situ." In Proceedings of the 2018 CHI Conference on Human Factors in Computing Systems, pp. 1-15. 2018. 
[26] Laghari, Asif Ali, Hui He, Asiya Khan, and Sajida Karim. "Impact of video file format on quality of experience (QoE) of multimedia content." 3D Research 9, no. 3 (2018): 39.

[27] Kim, Daehwan, and Yong Jae Ko. "The impact of virtual reality (VR) technology on sport spectators' flow experience and satisfaction." Computers in human behavior 93 (2019): 346-356.

[28] Laghari, Asif Ali, Hui He, Muhammad Shafiq, and Asiya Khan. "Application of quality of experience in networked services: Review, trend \& perspectives." Systemic Practice and Action Research 32, no. 5 (2019): 501-519.

[29] Saleme, Estêvão B., Alexandra Covaci, Gebremariam Assres, Ioan-Sorin Comsa, Ramona Trestian, Celso AS Santos, and Gheorghita Ghinea. "The influence of human factors on 360。 mulsemedia QoE." International Journal of Human-Computer Studies 146 (2020): 102550.

[30] Laghari, Asif Ali, Hui He, and Muhammad Ibrahim Channa. "Measuring effect of packet reordering on quality of experience (QoE) in video streaming." 3D Research 9, no. 3 (2018): 30.

[31] Pascoal, Rui, Ana De Almeida, and Rute C. Sofia. "Mobile Pervasive Augmented Reality Systems-MPARS: The Role of User Preferences in the Perceived Quality of Experience in Outdoor Applications." ACM Transactions on Internet Technology (TOIT) 20, no. 1 (2020): 1-17.

[32] Braud, Tristan, Farshid Hassani Bijarbooneh, Dimitris Chatzopoulos, and Pan Hui. "Future networking challenges: The case of mobile augmented reality." In 2017 IEEE 37th International Conference on Distributed Computing Systems (ICDCS), pp. 1796-1807. IEEE, 2017.

[33] Pallavicini, Federica, Alessandro Pepe, and Maria Eleonora Minissi. "Gaming in virtual reality: What changes in terms of usability, emotional response and sense of presence compared to non-immersive video games?." Simulation \& Gaming 50, no. 2 (2019): 136-159.

[34] Roettl, Johanna, and Ralf Terlutter. "The same video game in 2D, 3D or virtual reality-How does technology impact game evaluation and brand placements?." PloS one 13, no. 7 (2018): e0200724.

\section{Authors' Profiles}

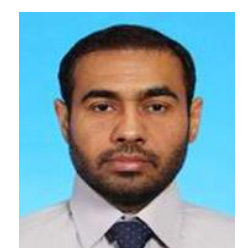

Asif Ali Laghari received the B.S. degree in Information Technology from the Quaid-e-Awam University of Engineering Science and Technology Nawabshah, Pakistan, in 2007 and Master degree in Information Technology from the QUEST Nawabshah Pakistan in 2014. From 2007 to 2008, he was a Lecturer in the Computer and Information Science Department, Digital Institute of Information Technology, Pakistan. In 2015, he joined the school of the Computer Science \& Technology, Harbin Institute of Technology, where he is now a PhD student. Currently he is Assistant professor in Sindh Madressatul Islam University, Karachi, Pakistan He has published more than 55 technical articles in scientific journals and conference proceedings. His current research interests include Computer networks, cloud computing, and multimedia QoE management.

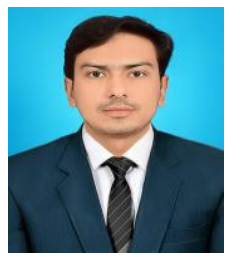

Awais K. Jumani received his BS(CS) and MS(CS) degrees from Shah Abdul Latif (SAL) University, Khairpur, Sindh, Pakistan, in 2014 and 2018. During his MS studies, Mr. Jumani was Teaching Assistant in Dept. of Computer Science at SAL University. Currently he is an Assistant Professor at ILMA University, Karachi Mr. Jumani has published over 26 Research Articles in scientific journals and conferences. His current interests include Machine Learning, Cloud Computing and Internet of Things.

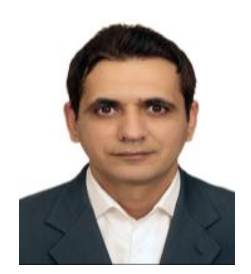

Dr. Kamlesh received his PhD in Computer Science and Technology from University of Electronic Science \& Technology of China (UESTC) in 2016. Currently, he is working as Assistant Professor in faculty of Information Technology at Sindh Madressatul Islam University, Karachi, Pakistan. His research interests include Image Processing, Computer Vision, Machine Learning, Knowledge Discovery and Data Mining, and Content Based Image Retrieval System.

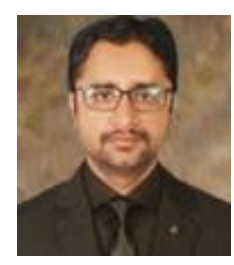

Mr. M.Ameen Chhajro obtained his MS degree in CSIT and he is doing PhD in Computer Science He has over 4 years of teaching and research experience. 
How to cite this paper: Asif A. Laghari, Awais K. Jumani, Kamlesh Kumar, M. Ameen Chhajro, " Systematic Analysis of Virtual Reality \& Augmented Reality", International Journal of Information Engineering and Electronic Business(IJIEEB), Vol.13, No.1, pp. 36-43, 2021. DOI: 10.5815/ijieeb.2021.01.04 